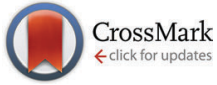

Cite this: Phys. Chem. Chem. Phys., 2014, 16, 22116

\section{The structure of deuterated benzene films adsorbed on the graphite (0001) basal plane: what happens below and above the monolayer coverage?}

\author{
Emanuel Bahn, ${ }^{\star a b}$ Holly Hedgeland, ${ }^{c}$ Andrew P. Jardine, ${ }^{b}$ Paul F. Henry, ${ }^{d}$ \\ Thomas C. Hansen ${ }^{a}$ and Peter Fouquet ${ }^{\star a}$
}

\begin{abstract}
An exact description of the interactions in aromatic carbon systems is a key condition for the design of carbon based nanomaterials. In this paper we investigate the binding and adsorbate structure of the simplest prototype system in this class - the single aromatic ring molecule benzene on graphite. We have collected neutron diffraction data of the ordered phase of deuterated benzene, $\mathrm{C}_{6} \mathrm{D}_{6}$, adsorbed on the graphite (0001) basal plane surface. We examined relative coverages from 0.15 up to 1.3 monolayers $(\mathrm{ML})$ in a temperature range of 80 to $250 \mathrm{~K}$. The results confirm the flat lying commensurate $(\sqrt{7} \times \sqrt{7}) R 19.1^{\circ}$ monolayer with lattice constants $a=b=6.5 \AA$ at coverages of less than $1 \mathrm{ML}$. For this structure we observe a progressive melting well below the desorption temperature. At higher coverages we do neither observe an ordered second layer nor a densification of the structure by upright tilting of first layer molecules, as generally assumed up to now. Instead, we see the formation of clusters with a

bulk crystalline structure for coverages only weakly exceeding $1 \mathrm{ML}$.
\end{abstract}

Received 29th July 2014,

Accepted 29th August 2014

www.rsc.org/pccp

\section{Introduction}

The structure of adsorbed benzene has been studied for several decades and yet continues to be of active interest. ${ }^{1,2}$ From a fundamental point of view, benzene is of great importance as it represents the smallest building block of a layered hexagonal carbon system and in spite of its high symmetry it gives rise to complex structures and dynamics. ${ }^{3-9} \mathrm{~A}$ detailed understanding of benzene physisorption is also important for the development and improvement of filtering systems: benzene is a highly toxic and carcinogenic aromatic hydrocarbon molecule that occurs naturally in crude oil and coal deposits and its safe removal is thus of great concern for petrochemistry. ${ }^{10,11}$ Finally, benzene on carbon surfaces has been considered to be an important prototype for studying elementary dynamic processes and friction in view of the development of nanometer size motorization systems. ${ }^{8,12}$

The structure of bulk crystalline benzene has been studied for more than fifty years. ${ }^{13-15}$ The benzene's multipole moments cause a perpendicular relative orientation of adjacent molecules

\footnotetext{
${ }^{a}$ Institut Laue-Langevin, CS 20156, 38042 Grenoble Cedex 9, France. E-mail: bahn@ill.fr, fouquet@ill.fr; Tel: +3347620 7204

${ }^{b}$ Cavendish Laboratory, J.J. Thompson Avenue, Cambridge, CB3 OHE, UK

${ }^{c}$ London Centre for Nanotechnology, University College London, London, WC1H OAH, UK

${ }^{d}$ European Spallation Source (ESS) AB, P.O. Box 176, 22100 Lund, Sweden
}

(the symmetry of the molecule suppresses the net charge and dipole moment). Recently, the structure of liquid benzene has also been investigated in detail. ${ }^{16}$ The sub-monolayer structure of benzene adsorbed on graphite has been the subject of much debate. Whilst authors using nuclear magnetic resonance (NMR) measurements ${ }^{3,17}$ and quasielastic neutron scattering (QENS) ${ }^{4}$ suggested that the molecules stood upright on the graphite surface, neutron diffraction studies suggested flat lying molecules and a $(\sqrt{7} \times \sqrt{7}) R 19.1^{\circ}$ commensurate monolayer structure. ${ }^{5}$ The flat adsorption geometry was confirmed several years later by low energy electron diffraction (LEED), ${ }^{18,19} \mathrm{X}$-ray diffraction, ${ }^{20}$ and further NMR measurements. ${ }^{21,22}$ These heterogenous results were also reflected in the theoretical work on benzene/graphite: the first molecular dynamics (MD) simulations produced a structure that consisted mainly of flat lying molecules, but also contained a non-negligible fraction of upright molecules. ${ }^{23-28}$ A recent Grand Canonical Monte Carlo (GCMC) simulation in the liquid phase (273-373 K) produced a monolayer consisting of mostly flat lying molecules. ${ }^{29}$ It is worth noting that in the latter publication including a quadrupole interaction in the calculation was shown to be of crucial importance. However, this is not surprising in view of the alternating structure of the benzene crystal that is mainly due to dominant quadrupole interactions. ${ }^{13}$ The existence of a second surface layer has been discussed on several occasions. Whilst, to our knowledge, 
no experiment before has aimed to study it, several simulations on this question have been performed..$^{23-26,28,29}$ The results differ, but suggest in their majority the existence of a disordered, liquid-like second layer. ${ }^{26,28,29}$ Finally, there has been a structural study using a density functional theory (DFT) approach. ${ }^{30}$ The latter does not consider, however, lateral interactions.

Here, we report on neutron diffraction measurements of fully deuterated benzene, $\mathrm{C}_{6} \mathrm{D}_{6}$, adsorbed on the basal plane surface (0001) of exfoliated graphite. Deuterium has to be used instead of hydrogen, as hydrogen scatters predominantly incoherently and, hence, contributes very little to the diffraction peaks. The measurements cover for the first time relative surface coverages between 0.15 and 1.3 monolayers (ML) in a coherent experiment. This represents a full overview of the structure from isolated molecules to the beginning of multi-layer formation. Our data indicate the formation of nano-clusters with a bulkcrystalline order at coverages of more than $1 \mathrm{ML}$. The structure was measured from low temperature across the melting transition up to $250 \mathrm{~K}$.

This paper is organised as follows: after this general introduction, we describe the sample preparation procedure and the instrument parameters used for the measurements. In the subsequent section, we present the experimental results and the analysis. We finish this paper with a discussion of the results in view of past results and future research.

\section{Experimental}

\subsection{Sample preparation}

Neutron and X-ray diffraction experiments of hydrocarbon molecules adsorbed on graphite require the use of high surface density substrates due to the small surface signal. Here, we used chemically exfoliated graphite, which is a widely used high surface density material that typically has a specific surface area of about $20 \mathrm{~m}^{2} \mathrm{~g}^{-1}$ and retains a sufficiently low defect density. ${ }^{31,32}$ In addition, exfoliated graphite samples show a preferential orientation of the basal plane surfaces. We exploited this and oriented the basal planes parallel to the scattering plane of the neutrons. We used $25 \mathrm{~g}$ of Papyex exfoliated graphite of grade N998 (>99.8\% C, Carbone Lorraine, Gennevilliers, France). We prepared exfoliated graphite disks of $2 \mathrm{~cm}$ diameter that were heated to $973 \mathrm{~K}$ under vacuum for 4 days and subsequently transferred to a cylindrical aluminium sample holder under a protective atmosphere. The sample holder was sealed by a lid with a steel knife edge and connected to a gas sorption system via a heated steel capillary. The surface density of the sample was measured in situ by BET (Brunauer-EmmettTeller) nitrogen adsorption isotherms that were obtained using a high precision absolute pressure gauge (Baratron 690A, MKS Instruments). We found a specific surface area of $23 \mathrm{~m}^{2} \mathrm{~g}^{-1}$. Fully deuterated benzene (99.6\% D, CEA Saclay, France) was used in this experiment to enhance coherent scattering. In this article we define the relative surface coverage, $\theta$, by the ratio of the molecular density, $\rho$, divided by the density of the $(\sqrt{7} \times \sqrt{7}) R 19.1^{\circ}$ structure $\left(\rho_{\mathrm{ML}}=1 / 36.7 \AA^{-2},{ }^{19}\right): \theta=\rho / \rho_{\mathrm{ML}}$.
The density, $\rho$, was given by the change in pressure of the benzene during adsorption (exploiting the finite vapor pressure of benzene at room temperature) and by the known surface area of the sample.

\subsection{Diffraction measurements}

Neutron diffraction measurements were carried out using the high intensity powder diffractometer D20 at Institut LaueLangevin (ILL), Grenoble, France, using a wavelength of $\lambda=2.4 \AA^{33}{ }^{33}$ Data were taken in a range of momentum transfers $Q=\left|\vec{k}_{\mathrm{f}}-\vec{k}_{\mathrm{i}}\right|=\left[0.2\right.$ to 5.1] $\AA^{-1}$, where $\vec{k}_{\mathrm{i}}$ and $\vec{k}_{\mathrm{f}}$ are the neutron wavevectors before and after scattering from the sample, respectively. The resolution in this set-up for a $2 \mathrm{~cm}$ diameter sample is about $\Delta Q / Q=0.03 .{ }^{33}$ Measurements were performed at relative surface coverages of $0.15 \mathrm{ML}, 0.5 \mathrm{ML}, 0.8 \mathrm{ML}$, and 1.3 ML, respectively, and at temperatures in the range [80 to 250] $\mathrm{K}$. The temperature was controlled using a standard liquid helium cryostat ('orange' cryostat, ${ }^{34}$ ). Additional diffraction measurements of the clean graphite sample were performed at all temperatures. The graphite substrate and its orientation remained the same throughout all measurements. Subsequently, the clean graphite data were subtracted from the diffraction data of the adsorbate systems at equal temperature (see Fig. 1). Five regions in our data show very strong signal from the graphite substrate that masks the signal from the benzene adsorbate and makes a meaningful interpretation of the benzene signal impossible. These regions, as well as the low $Q$ region, are marked by grey shading in Fig. 1 and excluded in our further data analysis.

\subsection{Neutron spin echo measurements}

Supplemental neutron spin echo (NSE) measurements were performed on the NSE spectrometer IN11 at ILL using the high signal set-up IN11C, which uses a 30 degree detector bank. ${ }^{35}$ Here, we used a wavelength of $\lambda=5.5 \AA$ for the maximum signal. The spectra were normalized using a standard procedure: all data were divided by a spectrum that was obtained in situ at the cryostat base temperature of $1.5 \mathrm{~K}$, where the system can safely be assumed to be static in the dynamic window of the

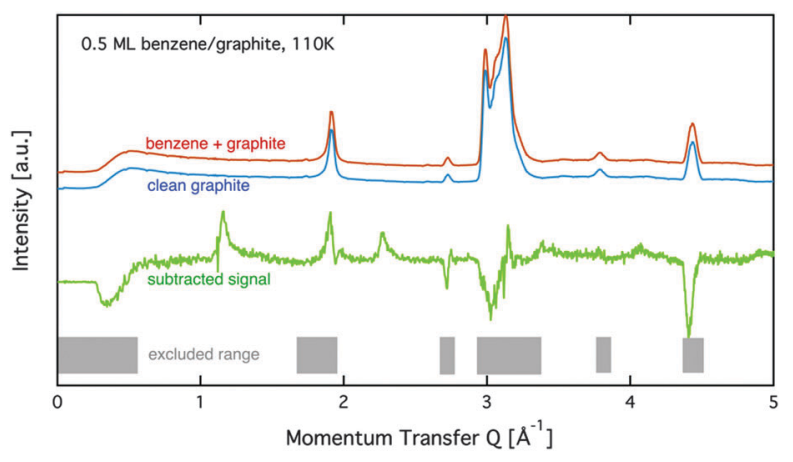

Fig. 1 Diffraction data at $110 \mathrm{~K}$ for the empty graphite sample and a coverage of $0.5 \mathrm{ML}$ are shown together with the subtracted data. Regions with a strong graphite signal are indicated by a grey pattern. These regions are omitted in the analysis in this paper. 
spectrometer. The experiment covered a range of momentum transfers of $Q=[0.2$ to 0.7$] \AA^{-1}$.

\section{Results and discussion}

Fig. 2 displays an overview of the coverage dependence of the diffraction patterns of benzene/graphite at a temperature of $110 \mathrm{~K}$. The graphs show significant structural differences between the sub-monolayer and the multi-layer regime as new peaks appear above 1 ML. Hence, for the sake of clarity of the description, we will describe these two regimes in separate sections.

\subsection{Diffraction data and structural refinement - sub-monolayer regime}

The diffraction pattern of the monolayer of benzene/graphite at low temperature has been studied many times before and there exists general agreement on a $(\sqrt{7} \times \sqrt{7}) R 19.1^{\circ}$ pattern with respect to the graphite (0001) surface structure both for $\mathrm{C}_{6} \mathrm{H}_{6}$ and $\mathrm{C}_{6} \mathrm{D}_{6} \cdot{ }^{5,18-22}$ However, the capabilities of neutron powder diffractometers and the corresponding data treatment methods have advanced tremendously since the last data were published and we can now study the structural changes upon heating up to the monolayer desorption temperature of $150 \mathrm{~K} .{ }^{36}$ In addition, the strong signal of the D20 diffractometer allows us to study several sub-monolayer coverages.

We start our analysis with the lowest measured coverage of $0.15 \mathrm{ML}$ (see Fig. 2). At 0.15 ML, no diffraction peaks are observed, which signifies that no long range order arises. The graphs of the two higher sub-monolayer coverages 0.5 ML and $0.8 \mathrm{ML}$ show four strong peaks outside of the regions that are dominated by graphite peaks. The benzene peaks remain at the same position up to $1 \mathrm{ML}$ coverage and have an asymmetrical shape that is typical for diffraction patterns of two-dimensional poly-crystalline systems. ${ }^{37,38}$ The asymmetric shape of the diffraction peaks is caused by the random orientation of crystallographic domains, where the normal of the domain remains parallel to the surface normal.

For a more detailed analysis of the sub-monolayer and monolayer structures we have simulated neutron diffraction

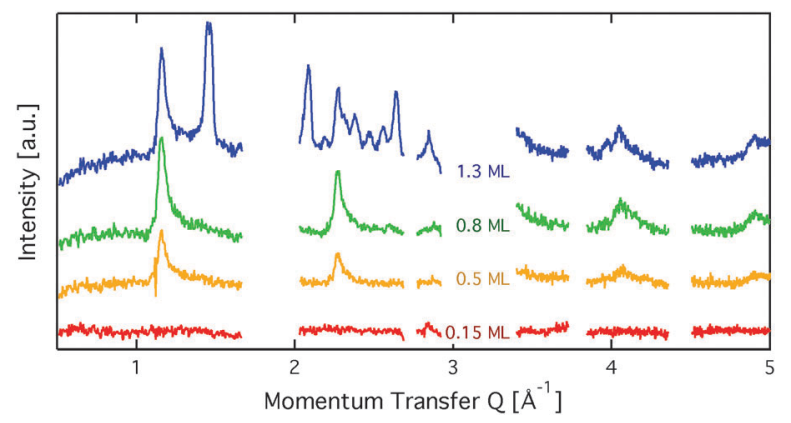

Fig. 2 Diffraction patterns for varying coverages at a sample temperature of $110 \mathrm{~K}$. Regions with strong graphite signal are omitted in the graph. At a coverage of $0.15 \mathrm{ML}$ no structural peaks are seen. At a coverage of more than $1 \mathrm{ML}$ additional peaks appear, which cannot be explained in the framework of a flat benzene structure.

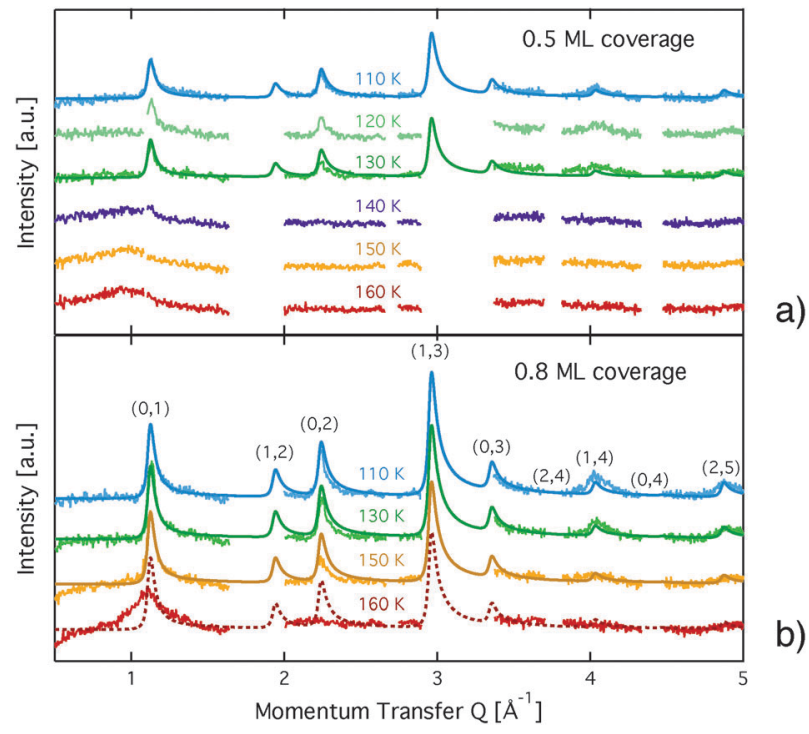

Fig. 3 Diffraction patterns for varying temperatures at a coverage of (a) $0.5 \mathrm{ML}$ and (b) $0.8 \mathrm{ML}$. The respective calculated diffraction patterns for $\mathrm{a}$ commensurate $(\sqrt{7} \times \sqrt{7}) R 19.1^{\circ}$ monolayer are shown by the solid lines. The pattern above $130 \mathrm{~K}$ at a coverage of $0.5 \mathrm{ML}$ and above $150 \mathrm{~K}$ at $0.8 \mathrm{ML}$, respectively, cannot be approximated by a $(\sqrt{7} \times \sqrt{7}) R 19.1^{\circ}$ structure and the corresponding expected pattern is, therefore, indicated by a broken line.

patterns of a flat lying monolayer using the software package nxpattern, which allowed us to adjust a number of parameters manually. ${ }^{39}$ The results of such a manual structural refinement are displayed in Fig. 3. The positions of the atoms in the deuterated benzene molecule were based on neutron diffraction data for deuterated benzene molecules. ${ }^{15}$ We have then adjusted the lattice parameters and found clear consistency with the model of a monolayer of densely packed flat lying benzene molecules. In the next step we have simulated results for out-of-plane tilting of molecules and in-plane rotations. We can exclude rotations of more than 1 degree and tilt of more than 10 degrees, due to the subsequent mismatch between the calculated and measured peak positions.

Further information could be drawn from the line-shape: in-plane neutron scattering from a flat, two dimensional layer of ordered islands that are randomly oriented produces diffraction peaks that drop sharply for lower angles and exhibit a Lorentzian decay for higher angles. ${ }^{37}$ Even better results were obtained in our case by using a squared Lorentzian peak shape that has been theoretically shown to be an indication of random layer lattices that undergo strain effects. ${ }^{40}$ The peak width of the diffraction pattern depends on the instrumental resolution $\Delta Q / Q$ and on the size of the islands. For a decreasing island size we find an increase of the width of the diffraction peak. For the $110 \mathrm{~K}$ data the width of the diffraction peaks was found to be equal to $\Delta Q / Q$. Therefore, we can consider the island diameter obtained from the simulations, $D=200 \pm 50 \AA$, a lower limit for the real island size. The graphite layers in the compressed exfoliated graphite exhibit a preferred orientation in the plane with a mosaic spread of the surface normal of 20-30 degrees 
Table 1 Measured and calculated positions of the diffraction peaks of the sub-monolayer structure at a coverage of 0.8 ML. All values are in units of $\AA^{-1}$

\begin{tabular}{lll}
\hline Index & Measured position & Simulated position \\
\hline$(0,1)$ & $1.124 \pm 0.011$ & 1.116 \\
$(1,2)$ & Hidden & 1.933 \\
$(0,2)$ & $2.220 \pm 0.022$ & 2.232 \\
$(1,3)$ & Hidden & 2.953 \\
$(0,3)$ & Hidden & 3.486 \\
$(2,4)$ & Hidden & 3.867 \\
$(1,4)$ & $4.042 \pm 0.040$ & 4.024 \\
$(0,4)$ & Hidden & 4.465 \\
$(2,5)$ & $4.894 \pm 0.049$ & 4.865
\end{tabular}

full width half maximum (FWHM) ${ }^{41,42}$ This also influences the peak shape and has been taken into account in the simulation of the pattern. Finally, the Debye-Waller factor, given by DWF = $\exp \left[-Q^{2}\left\langle u^{2}\right\rangle / 3\right]\left(\left\langle u^{2}\right\rangle\right.$ is the mean square displacement of the atoms) has a significant influence on the relative amplitudes of the measured peaks (see Fig. 3): the intensity of the diffraction peaks drops with increasing momentum transfer, $Q$.

The diffraction patterns resulting from the structural refinement for the coverages $0.5 \mathrm{ML}$ and $0.8 \mathrm{ML}$, respectively, are shown in Fig. 3. The agreement with the measured diffraction data confirms the formation of a $(\sqrt{7} \times \sqrt{7}) R 19.1^{\circ}$ structure up to a temperature of $130 \mathrm{~K}(0.5 \mathrm{ML})$ and $150 \mathrm{~K}(0.8 \mathrm{ML})$, respectively. The measured diffraction peak positions and the simulated peak positions in the $Q$-range of our data are compared in Table 1. The positions of the four available peaks are reproduced well within the experimental error bar, however we find a clear deviation of the intensities of the higher order peaks from the Debye-Waller approximation at temperatures approaching $150 \mathrm{~K}$ (Fig. 3). This is an indication of the gradual melting of the structure. The $(\sqrt{7} \times \sqrt{7}) R 19.1^{\circ}$ structure is depicted in Fig. 4. Here, we propose two possible variants. The structure in Fig. 4a assumes the positions of the benzene hydrogen atoms on top of the underlying carbon atoms. This structure has been preferred recently by other authors. ${ }^{30}$ In the structure of Fig. $4 \mathrm{~b}$ the hydrogen atoms are turned to take a maximum distance from the top layer carbon atoms. Since the scattering plane is concomitant with the surface planes our diffraction data cannot distinguish between the two orientations, but orientation (b) is preferred by our MD simulations, ${ }^{9}$ where the most probable orientation of the benzene rings with respect to the carbon structure is found to be turned by about $20^{\circ}$. An $I-V$ analysis of low intensity LEED measurements might resolve this issue, but it has to be kept in mind that the benzene islands remain highly dynamic even at temperatures of $60 \mathrm{~K}$ as shown by recent spectroscopy data. ${ }^{43}$ It would also be highly interesting to study theoretically whether many-body van der Waals interactions have an effect on the structure and phase transitions as these interactions have not yet been taken into account, to our knowledge. ${ }^{44}$

\subsection{Comparison to neutron spin-echo data}

Neutron spin-echo (NSE) spectroscopy measures the decay of structural correlation with time. NSE spectra for temperatures

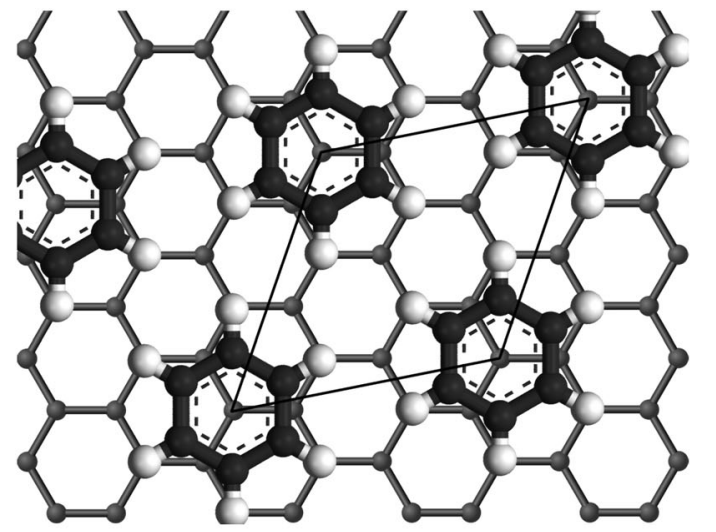

a)

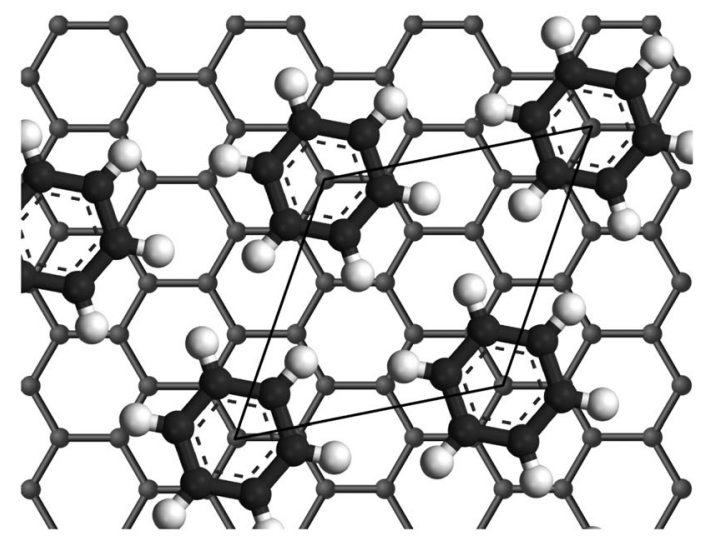

b)

Fig. 4 Illustrations of the commensurate $(\sqrt{7} \times \sqrt{7}) R 19.1^{\circ}$ structure. The hydrogen atoms are drawn in white, the benzene carbon atoms are black and the carbon atoms of the graphite substrate are shown in grey. (a) The hydrogen atoms are positioned on top of first layer carbon atoms. (b) The hydrogen atoms are turned away from the carbon atom positions. Orientation (b) agrees with our recent MD calculations. ${ }^{9}$

between 120 and $150 \mathrm{~K}$ are shown in Fig. 5. The NSE spectra show a decay that can be expressed by a simple exponential

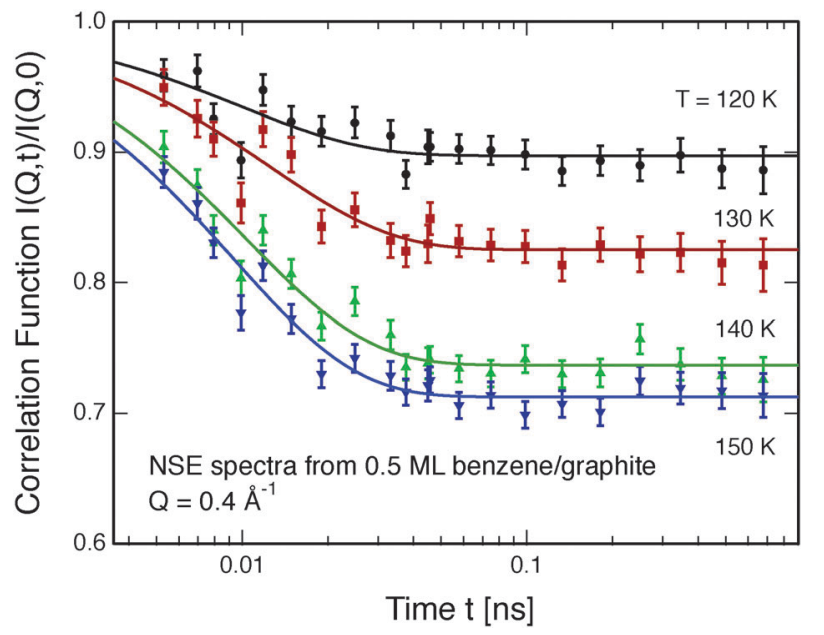

Fig. 5 Neutron spin echo spectra of a $0.5 \mathrm{ML}$ benzene layer on exfoliated graphite measured at temperatures between 120 and $150 \mathrm{~K}$ at a momentum transfer of $Q=0.4 \AA^{-1}$. The solid lines are exponential function fits to the data. 


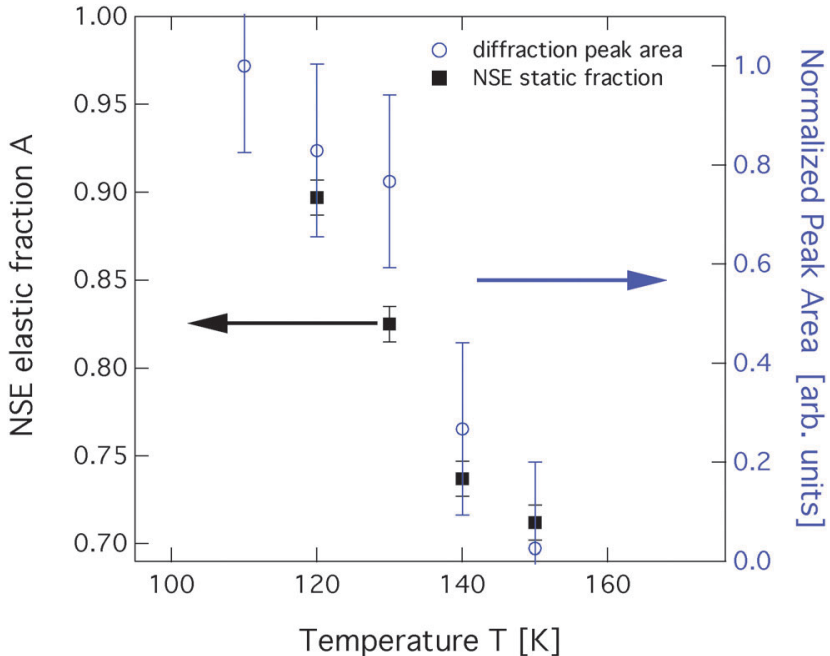

Fig. 6 Comparison of the total area of the $(0,2)$ diffraction peak (blue circles) to the static scattering $A$ parameter from NSE spectroscopy at a momentum transfer $Q=0.4 \AA^{-1}$ (black squares). Both data sets were obtained at a coverage of $0.5 \mathrm{ML}$.

decay that reaches a plateau value $A$ at large time: $I(Q, t) / I(Q, 0)=$ $(1-A) \times \exp [-t / \tau(Q)]+A$, where $\tau(Q)$ is the decay time of the structural correlation function. The level of the long time plateau $A$ depends strongly on the sample temperature. The plateau represents the scattering from the static fraction of the sample, i.e., from the graphite substrate and from immobile islands of benzene. The change in the level of the plateau indicates a gradual melting of the layer, which corroborates our interpretation of the decay of the signal of the higher order diffraction peaks. In Fig. 6 , we compare the area of the $(0,2)$ peak of the $0.5 \mathrm{ML}$ diffraction data set with the $A$ parameter extracted from fitting the NSE data at a momentum transfer $Q=0.4 \AA^{-1}$. From our diffraction and NSE data we can, thus, deduce that islands are formed at 0.5 and $0.8 \mathrm{ML}$ coverages below 140 and $160 \mathrm{~K}$, respectively, that are ordered in the $(\sqrt{7} \times \sqrt{7}) R 19.1^{\circ}$ monolayer structure. As the temperature approaches $140 \mathrm{~K}$ and $160 \mathrm{~K}$, respectively, we see a gradual melting.

\subsection{Diffraction results and structural refinement - coverage exceeding $1 \mathrm{ML}$}

Diffraction data for a coverage of 1.3 ML are shown in Fig. 7. Up to a temperature of $160 \mathrm{~K}$ additional peaks are clearly visible and the peaks that are found for the sub-monolayer structure remain. We have performed structural refinement by assuming either flat double layers or compressed layers with alternating flat and upright molecules in various geometries. None of these models reproduce any of the observed additional lines for the 1.3 ML coverage. Furthermore, we observed that the additional peaks that appear above 1 ML coverage (see Fig. 7) disappear at around $200 \mathrm{~K}$ and thus at a temperature that is $50 \mathrm{~K}$ above the monolayer desorption temperature. We conclude, that a second layer formation is not found here. Instead, we propose the formation of bulk crystalline benzene when the first layer is complete. In Fig. 7 we also compare our data with neutron

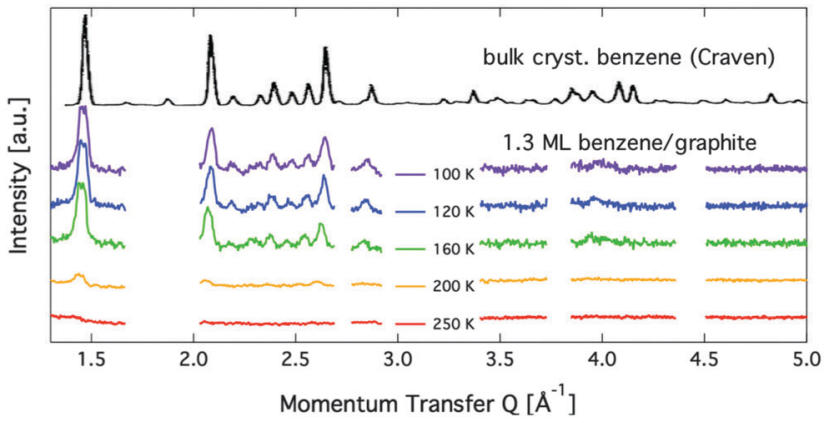

Fig. 7 Diffraction patterns for varying temperatures at a relative benzene coverage of $1.3 \mathrm{ML}$. The black line on top shows a neutron diffraction pattern from bulk benzene at $105 \mathrm{~K}$ that was originally obtained by Craven et al. ${ }^{14}$

diffraction data from (deuterated) bulk benzene by Craven $e t$ al. measured at the instrument D2B at ILL. ${ }^{14}$ The similarity to the bulk data provides clear evidence that the additional structure at above monolayer coverage is bulk crystalline benzene. We have no MD calculation data for coverages of more than $1 \mathrm{ML}$, but there is one more important indication that excludes a twodimensional structure: two-dimensional structures lead to strongly asymmetric peaks as observed for the sub-monolayer peaks, ${ }^{37,38}$ whereas the additional peaks in the $1.3 \mathrm{ML}$ data are clearly symmetric and cannot be formed from randomly oriented twodimensional ordered domains. The creation of the bulk structure at coverages with a relatively modest excess over $1 \mathrm{ML}$ is at odds with the widely spread picture of structure creation in the low coverage regime. ${ }^{45}$

\section{Conclusions}

We have performed neutron diffraction studies of deuterated benzene mono- and multilayers on exfoliated graphite. For a low coverage of $0.15 \mathrm{ML}$ no ordered structure was observed. For coverages between $0.5 \mathrm{ML}$ and $1 \mathrm{ML}$, the existence of a flat $(\sqrt{7} \times \sqrt{7}) R 19.1^{\circ}$ structure has been observed that can be shown to be caused by ordered islands of at least $200 \AA$ diameter. The ordered islands gradually melt at temperatures well below the desorption temperature and we find that the melting temperature changes with coverage. The islands disappear at $140 \mathrm{~K}$ for a coverage of $0.5 \mathrm{ML}$ and at $160 \mathrm{~K}$ for a coverage of $0.8 \mathrm{ML}$. The structure does not compress, within the experimental error bar, upon increasing the coverage. At a coverage of $1.3 \mathrm{ML}$, a bulk crystalline benzene structure appears rather than a second layer. This structure differs strongly from a compressed ordered structure suggested by other authors ${ }^{45}$ and indicates the formation of benzene nano-clusters in addition to an intact monolayer.

\section{Acknowledgements}

We would like to thank Dr Stuart Clarke and Adam Brewer for their elaborate help and their allocation of the structure analysis software. We would also like to thank Dr Hans Lauter for assistance with the adsorption system used in the neutron 
spectroscopy and diffraction studies. E.B. is grateful for financial support from the graduate school of the University of Grenoble.

\section{References}

1 S. Gokhale, P. Trischberger, D. Menzel, W. Widdra, H. Dröge, H.-P. Steinrück, U. Birkenheuer, U. Gutdeutsch and N. Rösch, J. Chem. Phys., 1998, 108, 5554-5564.

2 W. Liu, V. G. Ruiz, G.-X. Zhang, B. Santra, X. Ren, M. Scheffler and A. Tkatchenko, New J. Phys., 2013, 15, 053046.

3 B. Boddenberg and J. A. Moreno, Z. Naturforsch., A: Phys., Phys. Chem., Kosmophys., 1976, 31, 853-854.

4 R. Stockmeyer and H. Stortnik, Surf. Sci., 1979, 81, L315-L318.

5 P. Meehan, T. Rayment, R. K. Thomas, G. Bomchil and J. W. White, J. Chem. Soc., Faraday Trans. 1, 1980, 76, 2011-2016.

6 M. Monkenbusch and R. Stockmeyer, Ber. Bunsen-Ges., 1980, 84, 808-814.

7 P. Jakob and D. Menzel, Surf. Sci., 1989, 220, 70-95.

8 H. Hedgeland, P. Fouquet, A. P. Jardine, G. Alexandrowicz, W. Allison and J. Ellis, Nat. Phys., 2009, 5, 561-564.

9 P. Fouquet, M. R. Johnson, H. Hedgeland, A. P. Jardine, J. Ellis and W. Allison, Carbon, 2009, 47, 2627-2639.

10 D. C. Glass, C. N. Gray, G. G. Adams, R. W. Manuell and J. A. Bisby, Toxicol. Ind. Health, 2001, 17, 113-127.

11 M. T. Smith, Annu. Rev. Public Health, 2010, 31, 133-148.

12 W. R. Browne and B. L. Feringa, Nat. Nanotechnol., 2006, 1, 25-35.

13 E. G. Cox, Rev. Mod. Phys., 1958, 30, 159-162.

14 C. J. Craven, P. D. Hatton, C. J. Howard and G. S. Pawley, J. Chem. Phys., 1993, 98, 8236-8243.

15 G. A. Jeffrey, J. R. Ruble, R. K. McMullan and J. A. Pople, Proc. R. Soc. London, Ser. A, 1987, 414, 47-57.

16 T. F. Headen, C. A. Howard, N. T. Skipper, M. A. Wilkinson, D. T. Bowron and A. K. Soper, J. Am. Chem. Soc., 2010, 132, 5735-5742.

17 B. Boddenberg and J. A. Moreno, J. Phys., Colloq., 1977, 38, C4-52-C4-55.

18 U. Bardi, S. Magnanelli and G. Rovida, Surf. Sci. Lett., 1985, 165, L7-L11.

19 U. Bardi, S. Magnanelli and G. Rovida, Langmuir, 1987, 3, 159-163.

20 I. Gameson and T. Rayment, Chem. Phys. Lett., 1986, 123, 150-153.

21 B. Boddenberg and R. Grosse, Z. Naturforsch., A: Phys., Phys. Chem., Kosmophys., 1986, 41, 1361-1368.
22 R. Grosse and B. Boddenberg, Z. Phys. Chem., 1987, 152, 1-12.

23 C. Bondi and G. Taddei, Surf. Sci., 1988, 203, 587-593.

24 A. Vernov and W. A. Steele, Langmuir, 1991, 7, 2817-2820.

25 A. Vernov and W. A. Steele, Langmuir, 1991, 7, 3110-3117.

26 R. Hentschke and B. L. Schürmann, Surf. Sci., 1992, 262, 180-188.

27 M. A. Matties and R. Hentschke, Langmuir, 1996, 12, 2495-2500.

28 M. A. Matties and R. Hentschke, Langmuir, 1996, 12, 2501-2504.

29 N. Klomkliang, D. D. Do, D. Nicholson, C. Tangsathitkulchai and A. Wongkoblap, Chem. Eng. Sci., 2012, 69, 472-482.

30 S. D. Chakarova-Käck, E. Schröder, B. I. Lundqvist and D. C. Langreth, Phys. Rev. Lett., 2006, 96, 146107.

31 E. P. Gilbert, P. A. Reynolds and J. W. White, J. Chem. Soc., Faraday Trans., 1998, 94, 1861-1868.

32 C. Bockel, J. Coulomb and N. Dupont-Pavlovsky, Surf. Sci., 1982, 116, 369-379.

33 T. Hansen, P. Henry, H. Fischer, J. Torregrossa and P. Convert, Meas. Sci. Technol., 2008, 19, 034001.

34 D. Brochier, ILL Technical Report 77/74, Institut LaueLangevin technical report, 1977.

35 B. Farago, Physica B, 1998, 241-243, 113-116.

36 R. Zacharia, H. Ulbricht and T. Hertel, Phys. Rev. B: Condens. Matter Mater. Phys., 2004, 69, 155406.

37 B. E. Warren, Phys. Rev., 1941, 59, 693-698.

38 T. Arnold, S. Chanaa, S. M. Clarke, R. E. Cook and J. Z. Larese, Phys. Rev. B: Condens. Matter Mater. Phys, 2006, 74, 085421.

39 A. Y. Brewer, T. Friscic, L. M. Overvoorde, J. E. Parker, C. N. Richardson and S. M. Clarke, Mol. Phys., 2012, 111, 73-79.

40 S. Ergun and M. Berman, Acta Crystallogr., Sect. A: Cryst. Phys., Diffr., Theor. Gen. Crystallogr., 1973, 29, 12-17.

41 K. W. Herwig, J. C. Newton and H. Taub, Phys. Rev. B: Condens. Matter Mater. Phys., 1994, 50, 15287-15297.

42 T. Arnold, R. K. Thomas, M. A. Castro, S. M. Clarke, L. Messe and A. Inaba, Phys. Chem. Chem. Phys., 2002, 4, 345-351.

43 I. Calvo-Almazán, E. Bahn, M. M. Koza, M. Zbiri, M. Maccarini, M. T. F. Telling, S. Miret-Artés and P. Fouquet, Carbon, 2014, 79, 183-191.

44 V. V. Gobre and A. Tkatchenko, Nat. Commun., 2013, 4, 2341.

45 D. Do, D. Nicholson and H. Do, J. Colloid Interface Sci., 2008, $325,7-22$. 